\title{
Artelogie
}

Recherche sur les arts, le patrimoine et la littérature de l'Amérique latine

17| 2021

Transformaciones en Cuba contemporánea: cultura y sociedad

\section{Compte rendu de l'ouvrage de Roberto Cantú (ed.), Mexican mural art. Critical essays on a belligerent aesthetic, Cambridge, Cambridge scholars publishing, 2021, 281p.}

Élodie Vaudry

\section{OpenEdition}

\section{Journals}

Édition électronique

URL : https://journals.openedition.org/artelogie/9689

DOI : 10.4000/artelogie.9689

ISSN : 2115-6395

Éditeur

Association ESCAL

Référence électronique

Élodie Vaudry, « Compte rendu de l'ouvrage de Roberto Cantú (ed.), Mexican mural art. Critical essays on a belligerent aesthetic, Cambridge, Cambridge scholars publishing, 2021, 281p. », Artelogie [En ligne], 17 | 2021, mis en ligne le 07 septembre 2021, consulté le 15 septembre 2022. URL : http:// journals.openedition.org/artelogie/9689; DOI : https://doi.org/10.4000/artelogie.9689

Ce document a été généré automatiquement le 15 septembre 2022.

Tous droits réservés 


\title{
Compte rendu de l'ouvrage de Roberto Cantú (ed.), Mexican mural art. Critical essays on a belligerent aesthetic, Cambridge, Cambridge scholars publishing, 2021, 281p.
}

\author{
Élodie Vaudry
}

1 Roberto Cantú commence son introduction par une citation de l'écrivain mexicain Octavio Paz « lo poético no es algo que está fuera, en el poema, ni dentro, en nosotros, sino algo que hacemos y que nos hace. » (El arco y la lira, 1972 : 168). La "poétique » muraliste élaborée par les artistes mexicains, reflet de l'identité du Mexique postrévolutionnaire, est le fil conducteur tissé par Roberto Cantú et par les dix contributeurs de cette publication.

2 Issu d'un colloque international sur le muralisme mexicain organisé à l'université de Californie (Los Angeles) le 12 et 13 avril 2019, ce recueil d'essais ouvre sur des perspectives théoriques et critiques sur le sujet, mais également sur le contexte artistique, théorique, politique et historique inhérent au développement de cet engouement national et identitaire au Mexique après la Révolution de 1910. Bien loin de juxtaposer plusieurs facteurs liés à la conjoncture de l'art mural mexicain, ces écrits génèrent au contraire une dialectique basée sur les positions à la fois contrastées et fructueuses issues de cette riche conjoncture nationale et internationale. Le titre est explicite: "belligerent aesthetic»; il s'agit donc bien de mettre en regard des oppositions contre le goût européen et bourgeois, contre les régimes capitalistes et libéraux, contre encore les opposants au communisme de Staline, contre l'élitisme et enfin contre une identité mexicaine sclérosée par le joug post-colonial et dictatorial. De surcroît, la trame des belligérants se complexifie à mesure que les auteurs de ces essais interrogent ces résistances et mettent en lumière leurs apports plastiques et intellectuels pour le muralisme mexicain. Les antagonismes se rencontrent et 
nourrissent la scène artistique du Mexique de la première moitié du $\mathrm{XX}^{\mathrm{e}}$ siècle. Ces discours sur les dissensions se manifestent aussi dans la constitution même de l'ouvrage : la transversalité des approches - philosophie, histoire de l'art, littérature, cinéma, etc. -, la variété des thèmes abordés ainsi que l'internationalisme des auteurs suggèrent la complexité d'une thématique encore à explorer et la nécessité de "bousculer" la théorie liée au muralisme mexicain pour ré-activer des questionnements encore en suspends.

3 L'ouvrage se compose de 22 illustrations, dont un portrait de groupe des collaborateurs. Les reproductions, toutes en noir et blanc, déçoivent par leur petit nombre, mais la conjoncture de la pandémie ne facilitait pas l'accès aux droits de reproduction comme le précisent plusieurs auteurs. Afin de pallier ce manque, la référence à des sites en ligne pour accéder aux images est régulièrement présente en note et les descriptions ekphrasistiques aident le lecteur à suivre le discours et à s'immerger dans les œuvres étudiées. Les textes, destinés à un public d'anglophones et d'hispanophones, se clôturent par une courte biographie des participants afin d'appréhender au plus près leurs recherches sur le muralisme ou encore leurs liens, privés et/ou publics, avec la scène artistique mexicaine, voire latino-américaine.

4 La publication ouvre sur une introduction de Roberto Cantú, puis se découpe en trois parties. L'éditeur de l'ouvrage introduit le propos en immergeant le lecteur dans le contexte foisonnant, "glocal», transculturel et transnational du muralisme mexicain. Il commence par replacer ce recueil dans une bibliographie plus large caractérisée par des études idéologiques et historiques. Il insiste alors sur l'objectif de ces présents travaux qui est de proposer de nouvelles approches tout en ayant une réflexion critique sur l'historiographie existante du muralisme. L'introduction détaillée de Roberto Cantú donne le ton de l'ouvrage : la théorie de l'art, l'analyse critique des sources, la vie des artistes et la conjoncture nationale et internationale des œuvres représentent les éléments clefs de ces nouvelles appréhension du muralisme mexicain. Si les textes se centrent principalement sur les "trois grands », José Clemente Orozco, Diego Rivera y David Alfaro Siqueiros, c'est davantage pour développer leurs liens avec les processus politiques post-révolutionnaires au Mexique et aux États-Unis, avec les différentes ramifications du communisme et plus largement avec la conjoncture mondiale. L'auteur insiste également sur la nécessité d'étayer ce discours par les autres acteurs actifs sur la scène artistique du muralisme, tels que la photographe Tina Modotti, les peintres Rufino Tamayo, Jean Charlot et ceux de la Escuela al aire libre, etc.

5 Ensuite, la première partie "World Politics and Art in Revolutionnary Mexico", comprend trois articles de Renato González Mello, Alicia Azuela de la Cueva et Mary K. Coffey traitant des rapports contrastés entre les artistes muralistes mexicains et la politique du Mexique, mais également des États-Unis. Ces trois discours mettent en exergue l'inextricabilité du discours social et politique à celle de la lecture analytique du muralisme. En s'appuyant sur une historiographie qui court de 1920 avec les écrits des acteurs de cette époque tels que La raza cósmica (1925) de José Vasconcelos aux publications d'universitaires comme The Mexican Revolution (1986) d'Alan Knight, Renato González Mello, dans son essai "Propaganda and Muralism», croise étroitement l'élaboration des œuvres murales dirigées par la propagande et les divergences politiques des gouvernements mexicains successifs avec le regard qu'ont porté les acteurs et les historiens sur ces phénomènes artistico-politiques. Alicia Azuela de la Cueva, dans son étude "El Movimiento Artístico Revolucionario", revient sur 
cette dynamique de croisement en effectuant une périodisation du muralisme au regard des différents phases politiques de la Révolution de 1910 au " miracle mexicain » de 1940 à 1970. Cette chronologie précise met en exergue l'étroitesse des relations entre les artistes du mouvement et les politiques, et ainsi les fluctuations éthiques et esthétiques au gré des différents gouvernements. Ces recherches menées depuis de nombreuses années par l'auteur lui permettent aussi de questionner la notion d'« art public» attribuée au muralisme alors même que cette production est pensée par et pour les élites. Comme pour répondre à ces larges panoramas, l'article de Mary K. Coffey se concentre sur une aile du mural «The Epic of American Civilization » peinte en 1934 par José Clemente Orozco. L'analyse critique et précise de l'auteur met en relief la lecture du mythe de Quetzalcoatl par le peintre, ses positions vis-à-vis de l'idéologie pan-américaniste, économique et politique des États-Unis au sein du musée du Dartmouth College à Hanover.

6 La seconde partie "Muralism, Philosophy and the Critique of Visual Arts » regroupe quatre essais d'Octavio Armand, de Fernando Curiel Defossé, d'Héctor Jaimes et de Leonard Folgarait. Le lecteur commence cette section par s'échapper de la critique comme l'indique le titre du tableau peint par Pere Borrell del Caso (1835-1910) et analysé par Octavio Armand. Dans son article «Vano Azogue : de la extensión a la secuencia ", l'auteur entame une digression par la peinture du xix ${ }^{e}$ siècle, par la poésie, la littérature, la musique et la sculpture. Véritable Gesamtkunstwerk, cette essais effectue un détour par la prose de Shakespeare jusqu'à la réalité illusoire d'Alice dans le pays de Lewis Carroll. Sans aborder le muralisme, l'écrivain Octavio Armand intègre pourtant, par le biais de la métaphore, le lecteur dans le concept d'art total et d'effet miroir ; la fresque murale comme le passage vers une réalité recomposée, reflet d'une idée et d'un monde rêvé. Dans le second article «El Ateneo Muralista », Fernando Curiel Defossé poursuit ces parallèles avec les revues mexicaines contemporaines du muralisme et les écrits intimes des artistes, tels que ceux de José Clemente Orozco et Diego Rivera. Ainsi, en dehors de toute chronologie, l'auteur relate les controverses avec le Président Luis Echeverría dans les années 1970, les liens entre l'Académie, particulièrement le peintre Antonio Fabrés et les peintres Orozco et Rivera, ou encore présente les revues Savia Moderna (1906) et Revista Moderna (1898-1903) comme les antécédents intellectuels de la Révolution mexicaine et de l'idéologie du muralisme. Cette transdisciplinarité se tisse également sous la plume d' Héctor Jaimes dans son essai « Mexican Muralism : Between Art and Philosophy ». Principalement au travers des écrits - dont un inédit - de David Alfaro Siqueiros, l'auteur extrait les filaments philosophiques, comme les idées de Marx, Vasconcelos ou encore Engels, qu'il considère comme déterminants pour l'élaboration du muralisme mexicain. Pour clore cette seconde partie, Leonard Folgarait questionne le muralisme par le biais de la photographie et du cinéma, lui donnant ainsi une actualité étourdissante. L'auteur se fixe sur des détails de peintures murales de Diego Rivera et David Alfaro Siqueiros pour ensuite les projeter au sein d'un ensemble photographique de Tina Modotti et de Manuel Álvarez Bravo ou encore dans le film Roma d'Alfonso Cuarón. Cette étude phénoménologique met en parallèle les thématiques et les possibilités techniques de la photographie comme les distorsions, les flous, les écarts de point de vue avec les particularités des compositions muralistes.

7 La troisième partie « The Novels, Corridos, and Art Movements in Post-Revolutionary Mexico " regroupe trois articles de Georgina García Gutiérrez Vélez, Dafne Cruz Porchini et Iliana Alcántar. Ce dernier chapitre dresse de nouveau des liens étroits 
entre la structure et les attentes du roman et celles du muralisme, ou encore la trame esquissée par Diego Rivera entre l'URSS et le Mexique et enfin l'envers du muralisme avec les émouvants portraits des peintres María Izquierdo et Leonora Carrington. Georgina García Gutiérrez Vélez dans son essai «Carlos Fuentes, la Novela y el Muralismo : Puntualización » effectue une comparaison étonnante et très pertinente entre la construction narrative des écrits de Carlos Fuentes, notamment La región más transparente (1958) et les agencements plastiques du muralisme. L'auteur démontre aussi que les écrits de l'écrivain mexicain croisent les réalisations picturales par le biais des affinités politiques (le communisme), des thématiques socialistes portraiturées comme de véritables fresques de société. Cette étude démontre finalement comment Carlos Fuentes dialoguait avec son contemporain Diego Rivera, l'un avec sa plume l'autre avec son pinceau. Toujours dans l'esprit d'une dialectique transdisciplinaire, Daphné Cruz Porchini traite des sources de Diego Rivera dans son essai «The Visual Sources of Diego Rivera's Mural Corrido de la Revolución». De retour de l'URSS en 1928, Diego Rivera, avec son carnet de croquis en poche, élabore les fresques du Ministère de l'éducation publique. Photographiés par Tina Modotti, les esquisses réalisées par Rivera lors de son séjour soviétique sont présentées par l'auteur comme la base de son futur travail mexicain et surtout comme le début d'une longue discussion avec la mère du communisme. S'en suit alors l'analyse des échanges entre Rivera et le cinéaste soviétique Sergueï Eisenstein, et leur passion commune pour les aventures cinématographiques de Mickey Mouse et l'impact fondamental du film d'animation sur le peuple. La troisième et dernière partie se termine par les réponses féminines au muralisme mexicain étudiées par Iliana Alcántar dans son essai «María Izquierdo y Leonora Carrington: El otro movimiento artístico ». D'après les biographies des deux peintres écrites par Elena Poniatowska, l'auteur relate le désarroi de María Izquierdo face aux refus successifs des confrères masculins pour l'intégrer au groupe des muralistes. À la même époque, l'artiste anglaise Leonora Carington lutte au sein du groupe des surréalistes contre son compagnon d'un temps Max Ernst et contre une société patriarcale. Iliana Alcántar dresse, par l'entremise des mots de l'écrivaine Elena Poniatowska, les portraits intimes de deux femmes artistes dont la lutte révolutionnaire n'était pas celle pour la nation, mais bien celle pour exister artistiquement et socialement. L'auteur offre dans le même temps l'envers d'un groupe muraliste patriarcal et hermétique.

8 Tout au long de ces 281 pages, le lecteur, qui ne doit pas être trop néophyte pour s'immerger dans cet ouvrage, se retrouve face à deux ouvrages regroupés dans un seul livre; la présence récurrentes des notes l'invite à établir une double lecture et à s'ouvrir sur un champ de références bibliographiques pertinentes et souvent bien à propos pour approfondir l'étude. La lecture se fait d'autant plus complète que les auteurs se répondent et se citent entre eux. Bien au-delà de faciliter l'étude de l'ouvrage, cela montre surtout la cohérence de l'ensemble, la connaissance précise de leurs travaux respectifs ainsi que l'évolution de leur recherche dans une temporalité longue et fructueuse.

\section{SOMMAIRE}

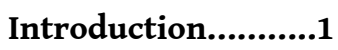

Part One : World Politics and Art in Revolutionary Mexico 


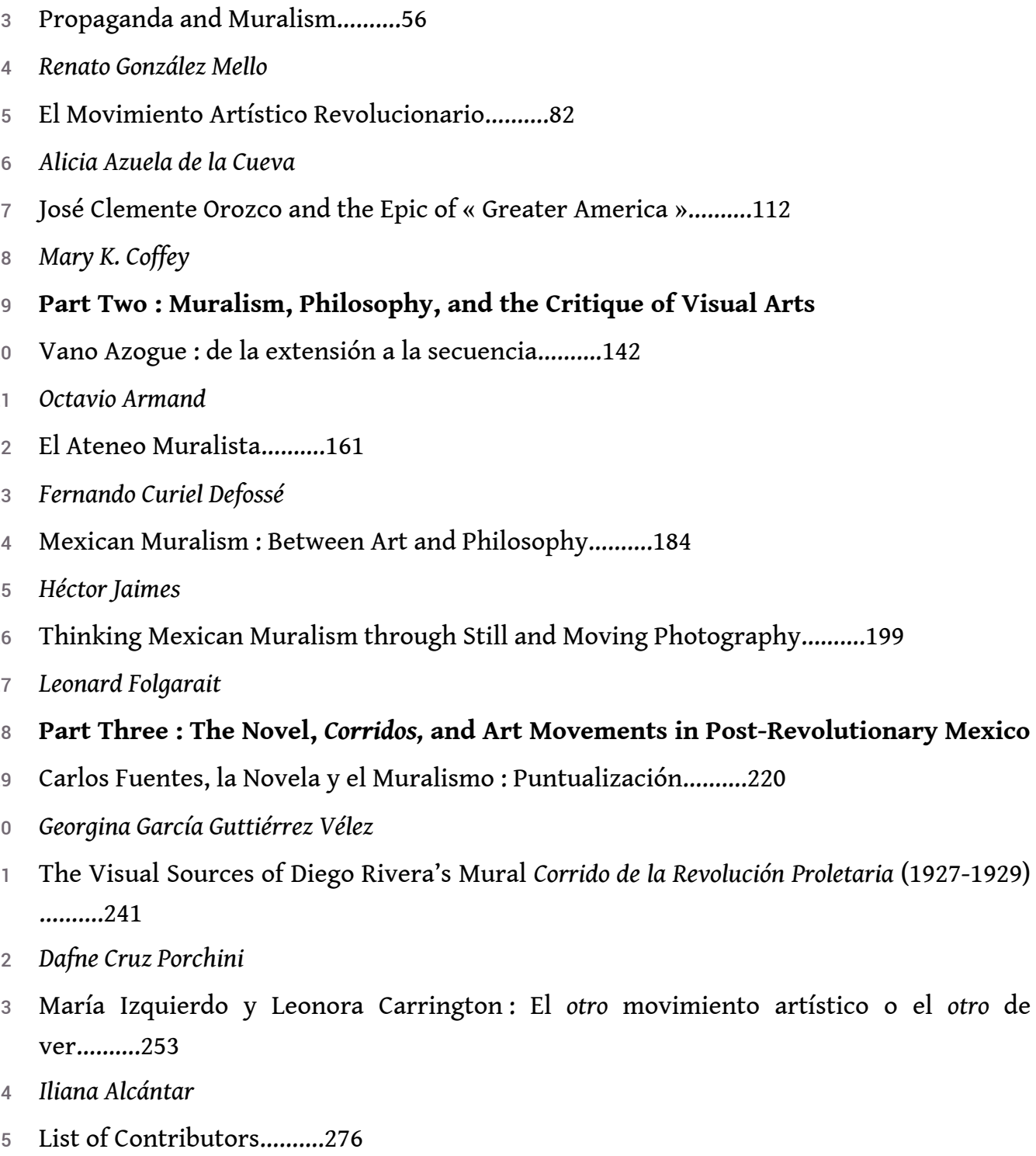

